\title{
Competencias emprendedoras en el aprendizaje aplicado al saber hacer
}

\section{$\mathbf{M}^{\mathrm{a}}$ Dolores Raigón ${ }^{\mathrm{a}}$ y Vicente Castell ${ }^{\mathrm{b}}$}

aDepartamento de Química. Universitat Politècnica de València. ETSI Agronómica y del Medio Natural (mail: mdraigon@qim.upv.es) y bepartamento de Producción Vegetal. Universitat Politècnica de València. ETSI Agronómica y del Medio Natural (mail: vcastell@ prv.upv.es)

\begin{abstract}
Practical work in the University courses on the development of the "entrepreneurial skills" allows promoting student potential capabilities in this aspect, and, at the same time, developing innovative skills. These training practices offer to future graduates the necessary prototypes to develop creation-processes of their own businesses. This contribution shows the obtained results in three subjects (two masters degrees and one degree with professional attributions) in relation to the entrepreneurial potential of students, who must deliver a practical work based on the development of an innovative product, having in mind the creation of a company. The developed product must be introduced to participants in the course, students and teachers, which will jointly evaluate it and thus, $95 \%$ of the final grade of the student in the subject. The main target is to enhance the innovative capacity of students and show the real evidence of entrepreneurial skills of them, whose development is encouraged through their own experience.
\end{abstract}

Keywords: creativity, entrepreneurial potential, product development, coevaluation

\section{Resumen}

El trabajo práctico en las asignaturas universitarias sobre el desarrollo de las "competencias emprendedoras" permite promover en el estudiante las posibles capacidades en esta faceta, a la par que desarrolla habilidades innovadoras. Estas prácticas formativas ofrecen los prototipos necesarios a los futuros egresados para desarrollar procesos de creación de sus propias empresas. La presente contribución expone los resultados obtenidos en tres asignaturas (dos de máster oficial y una de grado con atribuciones profesionales) en relación al potencial emprendedor de los estudiantes, que deben realizar un trabajo práctico basado en el desarrollo de un producto innovador, con miras a la creación de empresas. El producto desarrollado debe presentarse a los participantes en la asignatura, alumnos y profesores, que evaluarán conjuntamente el producto y, con ello, el 95\% de la nota final del alumno en la asignatura. El principal objetivo es potenciar la capacidad innovadora del estudiante y exponer la evidencia real de las competencias emprendedoras del alumnado, cuyo desarrollo se fomenta a través de la propia experiencia. 
Palabras clave: Creatividad, potencialidad emprendedora, desarrollo de producto, coevaluación.

\section{Introducción}

La formación universitaria se asume, de forma implícita, en la asimilación de un conjunto de contenidos científico-técnicos impartidos en las distintas asignaturas que configuran la titulación, y distribuidas en contenidos teóricos y prácticos, a los que se les asigna un valor en ECTS. Estos últimos repartidos en actividades de resolución de problemas, de prácticas de laboratorio, de campo e informáticas. Y todo ello diseñado bajo la consideración de una serie de valores competenciales y de responsabilidades en la formación de los futuros profesionales de la mayor excelencia.

Las competencias que se demandan en el mercado laboral actual no coinciden con la formación ofrecida desde las instituciones universitarias. Por ello, existe una necesidad de cambio de visión y misión en la enseñanza superior para ofrecer una cierta calidad y que se compensen las necesidades socio-económicas que solicita y valora positivamente el entorno empresarial, incluyendo habilidades y competencias, entre las que destaca la creatividad por parte de los estudiantes universitarios (González y Martínez, 2008).

Colás (2005) define la competencia como la "capacidad de los sujetos de seleccionar, movilizar y gestionar conocimientos, habilidades y destrezas para realizar acciones ajustadas a las demandas y fines deseados", por lo que la competencia debe materializarse en la sucesión de saberes, orientados a la producción, que deben articular una concepción del ser, del saber, del saber hacer y del saber convivir, es decir, el conjunto de conocimientos y capacidades adaptables que posee una persona para dar respuesta a una tarea de forma eficiente.

Las competencias referidas a capacidades creativas aluden a cómo las personas se enfrentan al trabajo en su conjunto, con la búsqueda de nuevas soluciones, generando ideas, asumiendo riesgos, innovando, aportando originalidad, conexión de ideas, entre otras muchas características. No obstante, para que el alumnado adquiera estas competencias se necesitan actividades académicas cuya finalidad sea la de facilitar su aprendizaje y herramientas que permitan su fomento y evaluación (Stevens y Levi, 2013).

Estas herramientas de aprendizaje deberán estar contextualizadas, implicar complejidad y estar focalizadas en el desarrollo, por parte de los estudiantes, de la capacidad de aplicación y resolución de problemas lo más reales posibles. En este enfoque el protagonista del proceso de aprendizaje es el propio alumno, mientras que el rol del profesor es, principalmente, acompañar, guiar, ser parte del proceso de evaluación, ayudar al alumno siempre que sea necesario (Fernández March, 2006). Estos elementos, además, desarrollan las competencias, ya que la concepción del aprendizaje como algo activo, individualizado y basado en el desarrollo cognitivo debe permitir al alumnado utilizar los conocimientos de manera creativa para resolver problemas reales (Villardón, 2006).

Dos herramientas de aprendizaje son básicas para garantizar el éxito: la metodología de aprendizaje y el sistema de evaluación. Es complicada la elección de una metodología de aprendizaje adecuada, ya que el binomio profesor-alumno es único, y el aprender haciendo

(cc)) EY-NC-ND 2016, Universitat Politècnica de València

Congreso IN-RED (2016) 
se debe ajustar a los objetivos y contenidos de la asignatura. Entre las diferentes metodologías de aprendizaje (Alcoba, 2012), el "estudio de casos" es una técnica en la que los alumnos analizan situaciones profesionales presentadas por el profesor, con el fin de llegar a una conceptualización analítica y exhaustiva y tiene como objetivo la capacitación práctica para la búsqueda de soluciones eficaces. Se trata de un sistema que es motivador, desarrolla la habilidad de análisis y síntesis, a la par que permite que el contenido sea más significativo para los alumnos y reflexionar con el grupo sobre los aprendizajes logrados. La evaluación del aprendizaje se plantea como un elemento estratégico en la universidad habida cuenta de la importancia que tiene la misma como elemento modulador del proceso de aprendizaje en cuanto que determina el qué, cómo, por qué y cuánto estudian los alumnos (Ibarra y Rodríguez, 2010). Entre los sistemas de evaluación que promueven el aprendizaje se encuentra la "evaluación de compañeros o evaluación entre iguales", que es útil porque permite que la evaluación sea parte del proceso de aprendizaje (aumentando la motivación del alumno al verse implicado en el proceso de evaluación), incrementa la responsabilidad y fomenta el espíritu crítico (Riesco y Díaz, 2007).

\section{Objetivos}

El principal objetivo del presente trabajo es mostrar los resultados obtenidos al aplicar un sistema novedoso en metodología de aprendizaje y evaluación en tres asignaturas, dos de máster oficial y una de grado con atribuciones profesionales, donde los alumnos deben realizar un estudio de caso práctico, basado en el desarrollo de un producto innovador, que será coevaluado de forma participativa por sus iguales.

Los objetivos paralelos son:

- Demostrar la capacidad creativa de los estudiantes en diferentes disciplinas.

- Motivar el potencial emprendedor de los estudiantes, en relación al aprendizaje, desarrollado con el trabajo.

- Tomar un papel más activo en el aprendizaje, capacitando al estudiante en la organización del tiempo, el trabajo metodológico, la observación, la habilidad para argumentar y defender sus decisiones, la competitividad, la capacidad para asumir errores propios, la capacidad dialéctica y la exposición oral.

- Retar al estudiante en la superación para alcanzar unos objetivos reales.

- Potenciar la responsabilidad y actitud crítica de los estudiantes, al tomar parte del proceso de evaluación.

\section{Desarrollo de la innovación}

La innovación docente ha consistido en la puesta en marcha en tres asignaturas de una metodología académica basada en una modificación del método "estudio de casos". Se trata de una modificación ya que los estudiantes combinan conceptos de esta metodología docente con el "aprendizaje orientado a proyectos", consistiendo el proyecto en el desarrollo de un producto real que deben diseñar y obtener de forma física, para ser evaluado por el conjunto de la clase.

(cc) EY-NC-ND 2016, Universitat Politècnica de València

Congreso In-Red (2016) 
Los criterios para el desarrollo de la actividad se explican al inicio del curso, de forma que el estudiante pueda ir aplicando los conceptos teóricos de la asignatura en el proyecto. Las pautas que se indican a los estudiantes en este punto son:

1.- Creación y desarrollo de un producto innovador dentro del sector productivo donde se encuadren los contenidos de la asignatura. El producto debe llevarse a la práctica y debe ser impactante a través del nombre (marca o registro), aprovechando recursos locales, técnicas tradicionales, etc.

2.- La idea del producto es individual y debe ser compartida, exclusivamente, con el profesor, el cual ayudará a resolver los posibles problemas técnicos en la elaboración. Para el resto de la clase debe mantenerse en secreto, hasta el momento de la exposición y evaluación.

3.- La evaluación del producto se realizará por el resto de los componentes de la clase, incluidos los profesores. El estudiante debe defender su propuesta, mediante exposición oral, mostrando físicamente el producto elaborado, para ser valorado, aplicando la técnica del test de Weiss (1981). Este test es una prueba objetiva donde el evaluador clasifica un atributo marcando una señal sobre la diagonal de un cuadrado de $10 \mathrm{~cm}$ de lado. La diagonal establece una escala desde mínimo (ninguna aceptación) hasta máximo (total aceptación) y la nota individual en cada atributo se consigue proyectando sobre la horizontal la marca señalada en la diagonal (figura 1), incluyéndose, además, un apartado con observaciones. Se valoran el total de los atributos, siendo uno de ellos la "apreciación global". El valor promedio obtenido en este apartado será el 95\% de la nota, el 5\% restante lo emite únicamente el profesor atendiendo a la memoria técnica presentada por el estudiante.

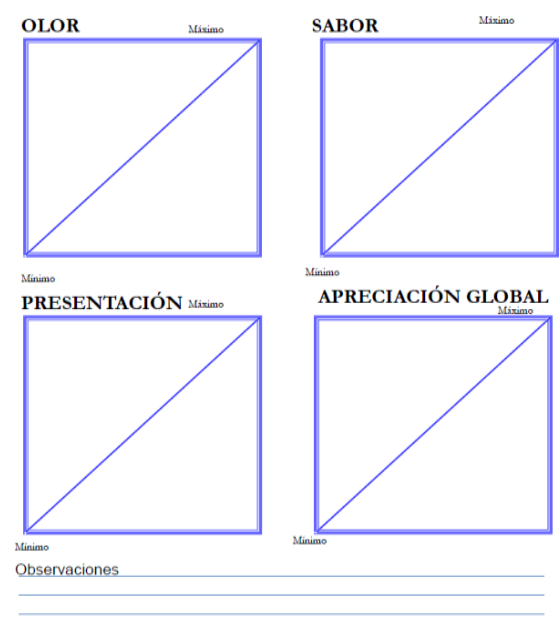

Fig. 1 Plantilla para la valoración de los atributos

Esta metodología de evaluación permite valorar el grado de aceptación del producto sin pensar en nota de forma numérica y, con ello, se evitan posibles predisposiciones en la calificación entre los pares. Con esta metodología el profesor asume el rol de experto y al 
finalizar cada valoración emite su criterio en público, indicando los posibles defectos, vacíos técnicos, las posibles mejoras al producto, etc.

La tabla 1 muestra la descripción del entorno donde se enmarcan las asignaturas implicadas en el estudio, las titulaciones a las que pertenecen, el número de alumnos y los cursos académicos de observación.

Tabla 1. Descripción de las asignaturas sometidas a innovación

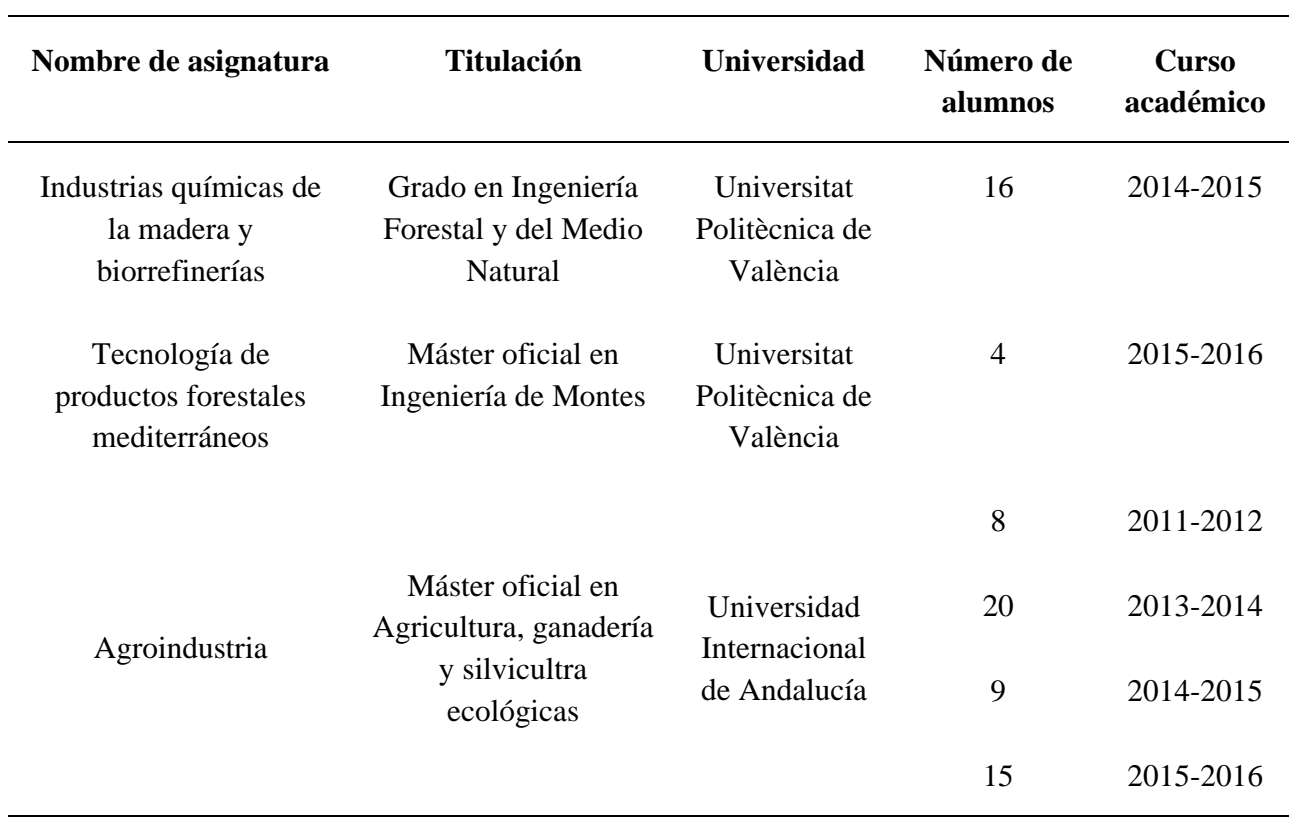

La asignatura "Industrias químicas de la madera y biorrefinerías" (código 11036) está incluida en la materia "Procesos industriales de los productos forestales" y tiene una carga docente de 6 ECTS. Se trata de una asignatura que deben cursar todos los estudiantes de la titulación en la intensificación de Industrias Forestales. El curso académico donde se realizó la innovación fue el 2014-2015 con 16 estudiantes matriculados, valorando en este caso sólo el atributo de "apreciación global". La asignatura "Tecnología de productos forestales mediterráneos" (código 33388) está incluida en la materia "Productos forestales" y tiene una carga docente de 6 ECTS. Se trata de una asignatura obligatoria en la especialidad de Industrias Forestales del máster universitario en Ingeniería de Montes que habilita para el ejercicio de la profesión de ingeniero de montes. El curso académico donde se realizó la innovación fue el 2015-2016 con 4 estudiantes matriculados (uno de ellos estudiante de programa de intercambio académico), valorando en éste los cuatro atributos. La asignatura "Agroindustria" (código 2104518) tiene una carga docente de 2 ECTS y está encuadrada en el módulo cuatro en el itinerario de "Agricultura". En esta asignatura es donde más se ha llevado a cabo la innovación con cuatro cursos académicos y en todos los casos se han evaluado los cuatro atributos. 


\section{Resultados}

Las asignaturas cuentan con un alto grado de heterogeneidad por lo que los resultados obtenidos se describen de forma individual para cada una de las mismas.

\subsection{Resultados de la asignatura "Industrias químicas de la madera y biorrefinerías"}

La respuesta de los estudiantes a la innovación fue muy positiva. Las observaciones generales son: 1) sólo un estudiante no se veía capaz de su ejecución, por falta de ideas y dedicación. El trabajo del profesor, en este caso, fue el de aportar ideas, información y pautas en la organización del tiempo, para poderlo desarrollar. 2) Cinco estudiantes realizaron un trabajo experimental para comprobar la respuesta técnica del producto y el resto realizó el trabajo autónomo, contando con el asesoramiento del profesor. 3) Dos estudiantes iniciaron los trámites para poder patentar el producto. 4) Un estudiante inició la comercialización del producto vía on-line y de proximidad. 5) Un estudiante ha mejorado su proceso para iniciar trámites de producción y comercialización. 6) Un estudiante va a llevar su prototipo para el desarrollo del trabajo final de grado.

El conjunto de propuestas presentadas tienen una amplia diversidad de ideas: 1) Ceniza para la produccion de lejía, jabón de potasa e insecticida ecológico; 2 ) "Resinizarte": uso de resina natural para la elaboración de artículos de ornamentación; 3) "Forestable": diseño de una mesa con utilidad de vivero en cajón inferior; 4) "Veraturia": diseño de una gama de productos naturales cosméticos terapéuticos, en concreto con propiedades para el tratamiento de huesos, cardiovasculares e hidratantes; 5) "Wood plastic": bioplásticos y polímeros a partir de materias primas orgánicas naturales; 6) "Lentiscus": extracto de las plantas con propiedades antifúngicas a partir de las hojas; 7) "On my own": cajas decorativas realizadas a partir de papel usado, elaborando láminas de papel y empleando colorantes naturales; 8) Ambientador fabricado exclusivamente con productos maderables, madera, corcho y unas varitas difusoras de madera; 9) "Aquaslide": producto impregnable en papel o tejidos que reproduzca las propiedades del efecto loto, repeliendo el agua, utilizando componentes de origen natural; 10) "Helios": sistema de generación energética de forma renovable, haciendo uso de la energía solar a nivel terrestre, en forma de vapor; 11) "Naturshave": tónicos en base a extractos de plantas aromáticas de acción calmante y refrescante; 12) "Manu-Z": prototipo de maceta doméstica con subproductos forestelas y sistema eléctrico e hidráulico, en el que se combinen las técnicas de cultivo hidropónico y aeropónico; 13) "EFIX": instalación de caldera de biomasa de restos forestales y agrícolas; 14) "Bamsty": bolígrafos recargables, elaborados con bambú y tinta ecológica; 15) "ECOFUNGHY": Kit de producción doméstica de setas y hongos comestibles; 16) "Hidromiel": bebida alcohólica de baja graduación en base a miel.

Aunque la idea de cada estudiante debía mantenerse en secreto, el compañerismo y la ilusión de la clase generó que se compartieran los prototipos sin eliminar el elemento sorpresa en el momento de la exposición. Todas las notas promedio superaron la calificación de notable (oscilando entre los valores de 7 y 9,2). Al comparar las notas promedio con las calificaciones individuales del profesor, se observa que en 8 casos la nota del profesor es superior a la del promedio de la clase con variaciones que oscilan entre el $1,5 \%$ y el $16 \%$, en un caso la nota promedio coincide con la del profesor y en 7 casos la nota del profesor es inferior a la del promedio de la clase, con variaciones que oscilan entre el $4,5 \%$ y el $18,7 \%$.

La figura 2 muestra algunos de los ejemplos de los productos, prototipos, efectos y marcas de los productos presentados en esta asignatura.

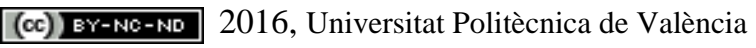




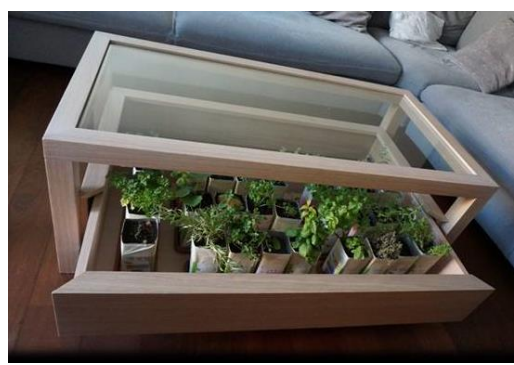

Forestable

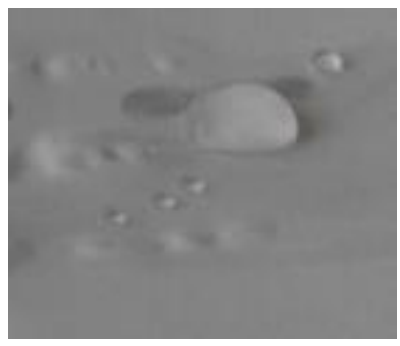

Efecto de Aquaslide sobre papel

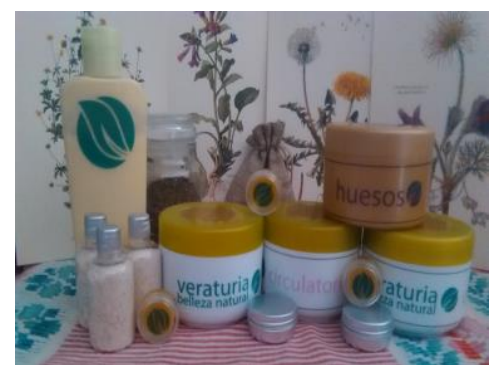

Veraturia

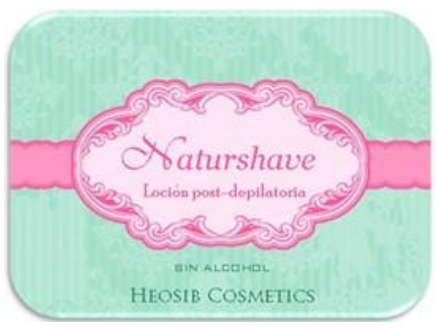

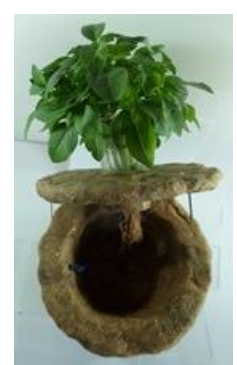

Мапи-Z

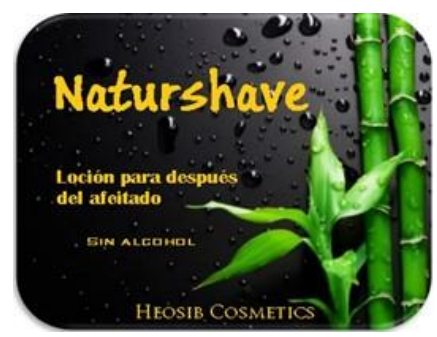

Etiquetas de Naturshave

Fig. 2 Ejemplos de productos, prototipos, efectos y marcas presentadas en la asignatura "Industrias químicas de la madera y biorrefinerías"

\subsection{Resultados de la asignatura "Tecnología de productos forestales mediterráneos"}

La asignatura "Tecnología de productos forestales mediterráneos" está compartida y dividida en cuatro partes y la innovación docente se lleva a cabo en una de ellas. Se puso de manifiesto la categoría de post-graduados de los estudiantes, ya que todos realizaron un trabajo autónomo. El profesor solamente realizó un asesoramiento técnico durante el proceso de elaboración. Para los cuatro estudiantes la innovación representó un reto ilusionante, pero con capacidad para llevarse a cabo.

En esta asignatura los productos debían ser una transformación alimentaria, realizada con productos forestales y mediterráneos. Los productos presentados fueron 1) Pan con frutos del bosque: elaboración panaria con arándanos, que proporciona un atractivo color morado; 2) Mermelada de Boletus: mermelada dulce de cuatro setas comestibles (Agaricus bisporus, Pholiota nameko, Pleorotus ostreatus y Lentinula edodes); 3) "Españoli": un nuevo concepto de aliño, atractivo y divertido formado por aceite de oliva virgen extra macerado con azafrán (resultando un aceite de color amarillento) y aceite de oliva virgen extra macerado con tomates secos y pimienta roja en polvo (resultando un aceite de color rojo), y presentación en pack que al presionar impulsa la combinación de los dos colores; 4) Jarabes con miel: caramelos de miel de eucalipto y falsa acacia, condimentados con jengibre, frambuesas, clavo y limón.

La figura 3 muestra algunos ejemplos de los productos presentados en la asignatura "Tecnología de productos forestales mediterráneos".

(c)) EY-NC-ND 2016, Universitat Politècnica de València

Congreso In-Red (2016) 


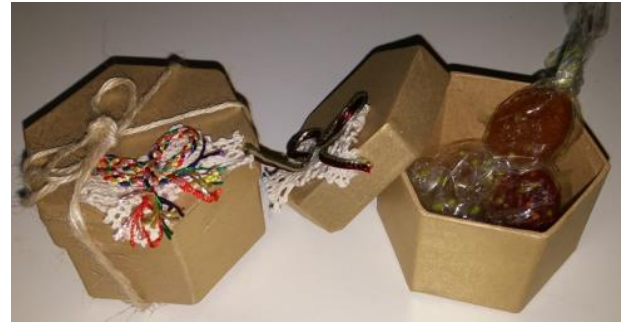

Jarabes con miel



Españoli

Fig. 3 Ejemplos de productos en la asignatura "Tecnología de productos forestales mediterráneos"

La figura 4 muestra los valores promedio de cada uno de los atributos estimados (presencia, sabor, textura y apreciación global) en cada uno de los productos, mientras que la línea negra (y etiqueta de datos) muestra la nota otorgada por el profesor al atributo de apreciación global a cada producto. En todos los casos la nota promedio de la clase fue superior a la del profesor, aunque las variaciones oscilan entre el $0,7 \%$ y el $6,7 \%$, lo que significa que en todos los casos están muy próximas.



Fig. 4 Valores para cada atributo por producto y nota del profesor (línea negra y valores) para los resultados de la asignatura "Tecnología de productos forestales mediterráneos"

\subsection{Resultados de la asignatura "Agroindustria"}

En la asignatura "Agroindustria" los estudiantes reciben formación sobre las diversas industrias de procesado de alimentos certificadas como ecológicas. Por tanto, el diseño de nuevos productos alimentarios tiene como objetivos a alcanzar el que sean innovadores, cumplan con parámetros de seguridad alimentaria, técnicas ecológicas y valor nutritivo. Para ello, los estudiantes reciben a lo largo del curso toda la formación técnica. En todos los cursos académicos los estudiantes han mostrado gran interés y han asumido el reto con un alto nivel técnico e innovador. La descripción exhaustiva de los 52 productos elaborados en los cuatro cursos académicos es inabordable en este trabajo, por lo que se mostrarán los 
resultados globales y los casos más destacados, así como las observaciones más importantes en cuanto a las notas obtenidas.

En el curso 2011-2012 se presentaron tres proyectos de mermeladas, uno de elaboración de pan de romero, un paté de aguacate y aceitunas (salado), una crema de aguacate y chocolate (dulce), galletas de avena con algarroba, elaborados lácteos en combinación con cereales de alto valor proteico y yogurt con frutas confitadas. Entre los casos presentados, un estudiante ha emprendido actividad empresarial y, en la actualidad, se encuentra con una pequeña empresa local que comercializa el producto presentado y cinco más en un comercio local y bajo la certificación ecológica europea. Otro estudiante tomó la idea para llevar a cabo su proyecto final de máster.

Se valoraron cinco atributos (presentación, olor, color, sabor y apreciación global). La figura 5 muestra la distribución radial de las notas otorgadas en promedio en cada atributo observado. Excepto en dos casos, donde el color y la presentación se valoraron más bajos, el resto de los productos tuvieron calificaciones uniformes.

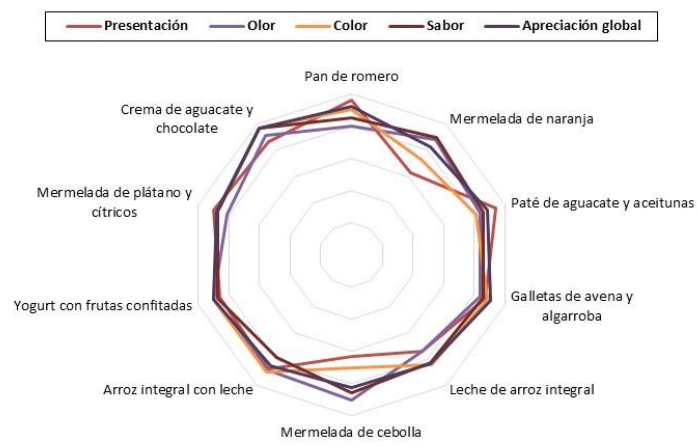

Fig. 5 Distribución radial de las notas para cada atributo por producto de la asignatura "Agroindustria” curso académico 2011-2012

El curso académico 2013-2014 destacó por ser muy creativo. Cuatro proyectos contaron con innovaciones de gran interés, como el "Letuario de naranjas": recuperación de un alimento de la España de los siglos XVI y XVII (Domingo, 1981); "Delicias de algarroba": galletas y dulces de algarroba; "La Cazoletera": línea de golosinas y helados ecológicos y una línea de "Alimentos infantiles ecológicos". No hay registro de las proyecciones empresariales que han podido desarrollar los estudiantes, pero el "Letuario" se proyectó como una posibilidad de reintroducir un alimento de alta apreciación organoléptica en los mercados gourmets.

La figura 6 muestra los valores promedio de cada uno de los atributos estimados (olor, sabor, presentación y apreciación global) en cada uno de los productos, mientras que la línea negra muestra la nota otorgada por el profesor al atributo de apreciación global a cada producto. Los alumnos valoraron 11 productos por encima del valor del profesor con incrementos que oscilan entre el 2,4\% y el 35,9\%, un producto se valoró a la par por el total de la clase y por el profesor en particular, y en ocho productos el profesor valoró por encima del promedio de la clase, oscilando los incrementos entre el 4,1\% y el $19 \%$.

(cc)) EY-NC-ND 2016, Universitat Politècnica de València

Congreso In-Red (2016) 


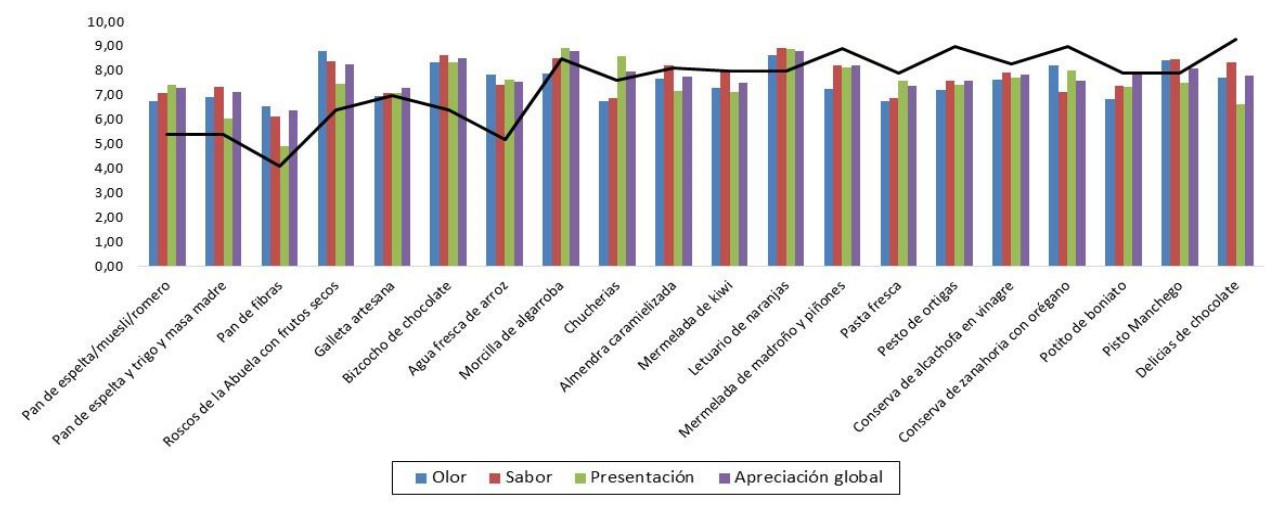

Fig. 6 Valores para cada atributo por producto y nota del profesor (línea negra) para los resultados de la asignatura “Agroindustria” curso académico 2013-2014

El curso académico 2014-2015 destacó porque se presentó el producto (proyecto) que mayor calificación ha obtenido en esta asignatura. Se trata de "Ecomelado", un producto dulce elaborado a partir de miel pura de mil flores, nueces y piñones ecológicos. Un estudiante ha llevado a cabo el desarrollo del producto a través de una empresa familiar y otro estudiante ha emprendido la producción a través de una asociación de consumo.

La figura 7 muestra los valores promedio de cada uno de los atributos estimados (presentación, sabor, textura y apreciación global) en cada uno de los productos, mientras que la línea negra muestra la nota otorgada por el profesor al atributo de apreciación global a cada producto. En seis casos el profesor ha valorado por encima del promedio de la clase, con variaciones que oscilan entre el 2,3\% y el 14,5\%, en dos casos la nota es similar y en un único caso el profesor valoró por debajo de la nota promedio de la clase.

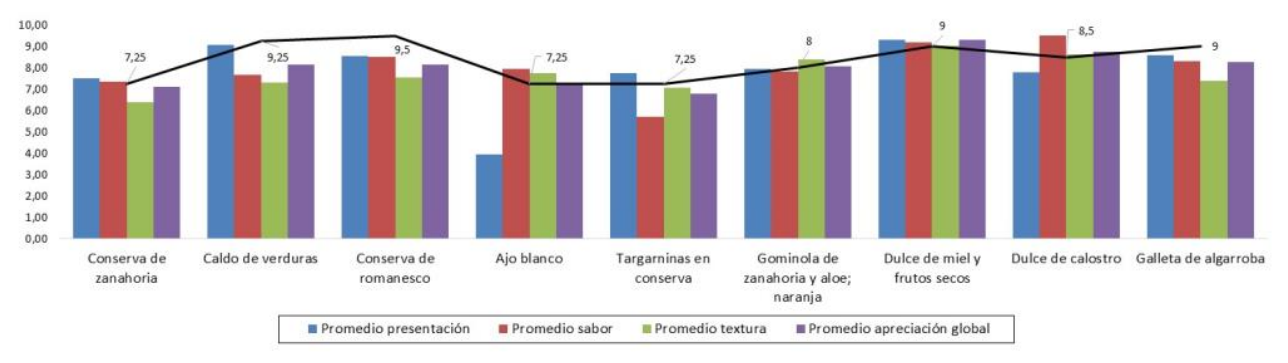

Fig. 7 Valores para cada atributo por producto y nota del profesor (línea negra) para los resultados de la asignatura “Agroindustria” curso académico 2014-2015

En el presente curso académico 2015-2016, los datos existentes manifiestan que un estudiante pretende llevar a cabo el proyecto como opción empresarial on-line y otro estudiante lo va a llevar a cabo dentro de un grupo de consumo, mientras que otro estudiante, que profesionalmente está vinculado a un colegio de personas discapacitadas como tutor, realizó su producto con el colectivo de educandos que tutela, resultando el proyecto doblemente atractivo, ya que sus alumnos se implicaron en todo el proceso desde la recolección hasta la esterilización de las conservas. Cada uno de sus alumnos pudo llevarse un frasco de la elaboración, contribuyendo a la mayor asertividad de la innovación. 
La figura 8 muestra los valores promedio de cada uno de los atributos estimados (olor, sabor, presentación y apreciación global) en cada uno de los productos, mientras que la línea negra muestra la nota otorgada por el profesor al atributo de apreciación global a cada producto. De todos los resultados de esta asignatura, en este curso académico es donde los estudiantes han sido más críticos con la evaluación, ya que, por una parte, todas las evaluaciones se proyectan sobre una nota, que en pocos casos supera el valor de 8 , y por otra parte, la valoración del profesor supera en todos los casos, excepto en uno que coincide, con la emitida en promedio por el total de la clase. Las variaciones entre la nota emitida por el profesor y el promedio de la clase son considerables, oscilando entre el 5,8\% y el $19,6 \%$.



Fig. 8 Valores para cada atributo por producto y nota del profesor (línea negra) para los resultados de la asignatura "Agroindustria” curso académico 2015-2016

La figura 9 muestra algunos ejemplos de los productos presentados en la asignatura "Agroindustria", así como una imagen de un momento de la evaluación, con la puesta en escena del producto antes de ser evaluado, mientras el estudiante autor del proyecto está realizando su exposición oral y mostrando el plan de viabilidad para la puesta en marcha de la posible empresa. En este momento, el resto de los estudiantes puede fotografiar el producto. Acabada la exposición, se abre el producto y se completa la valoración y la ficha de evaluación. Todos los estudiantes pueden opinar sobre el producto y sus atributos. El profesor obligatoriamente emite un juicio público y técnico sobre las características y posibilidades de mejora por ejemplo, en el caso del embalaje del producto "Cañade Pa", se recomienda una mejora cambiando el formato de vidrio por cazuela de cerámica, que aportaría mayor valor añadido al producto final.

Cualquier aportación al producto evaluado se realiza una vez se ha cumplimentado cada uno de los atributos a evaluar para no influir sobre el resto de estudiantes en el sentido de una mayor o menor apreciación del producto. Con ello, se facilita la sistematización de las observaciones y conclusiones que se derivan de la propuesta defendida por cada estudiante, proponiendo mejoras y haciendo observaciones que, incluso, puedan compartirse con toda la clase y/o con otros profesores. 


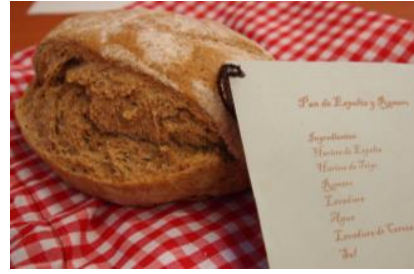

Proyecto panario (2011-2012)

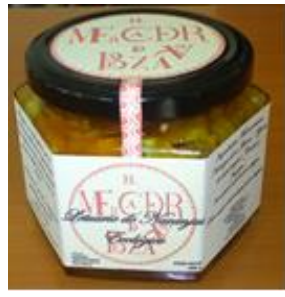

Letuario (2013-2014)

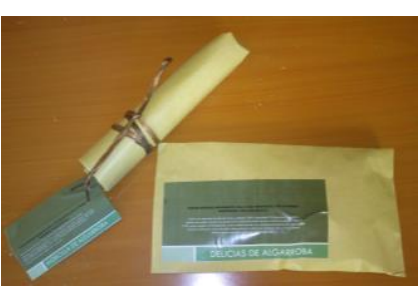

Delicias de algarroba (2013-2014)
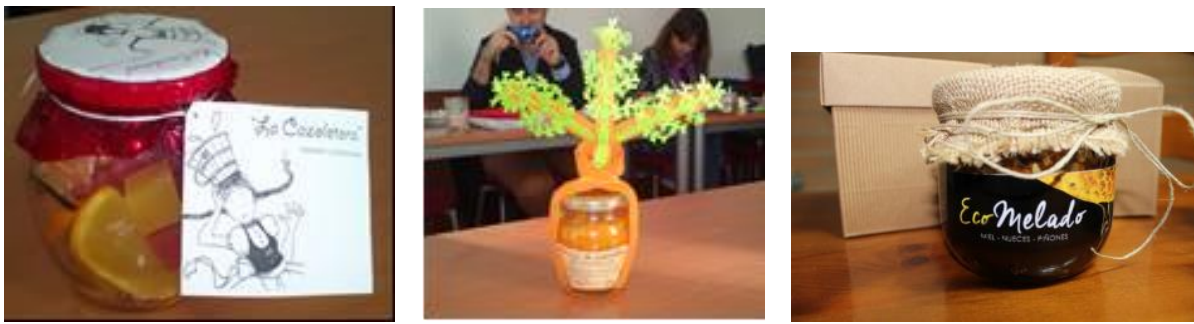

La Cazoleta: golosinas (2013-2014) Evaluación (2013-2014)
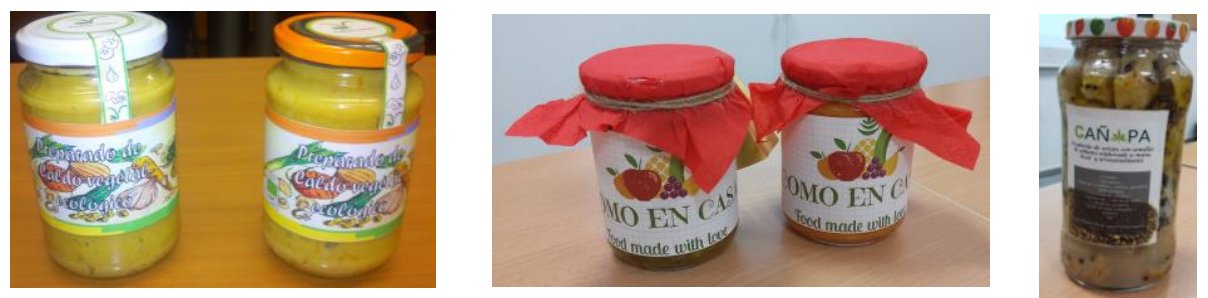

Concentrado de caldo vegetal (2014-2015)

CañadePa: seitán escabechado (2015-2016) Como en casa: alimentación tradicional (2015-2016)

Fig. 9 Ejemplos de productos en la asignatura "Agroindustria" en diferentes cursos académicos

En todas las asignaturas, la ejecución de la innovación metodológica ha sido posible porque el número de estudiantes por aula es bajo, ya que el sistema de evaluación requiere de tiempo en la exposición, en la valoración y en la fase de emitir criterios en público, pero crea un ambiente de grupo muy interesante desde el punto de vista social.

\section{Conclusiones}

Las principales conclusiones obtenidas del presente estudio son:

1. La introducción de la innovación metodológica y el afrontar el reto académico propuesto por el profesor ha permitido desarrollar y llevar a la práctica un amplio rango de innovaciones, mostrando un gran talento de todos los estudiantes. Este cambio en la orientación formativa permitirá a los estudiantes que puedan llegar a ser profesionales reflexivos, creativos y con una sólida base de conocimientos científicos y técnicos, basados en el saber hacer.

2. Las diferencias en la valoración y calificación emitida por el profesor y por el promedio de la clase no sufren grandes variaciones. Cuando el grupo es reducido, existe mayor concordancia entre las dos notas, cuando el grupo es numeroso se aprecian las mayores desviaciones entre las dos notas. En la gran mayoría de los 
casos, los estudiantes son más críticos con la apreciación y emisión de criterio que el propio profesor.

3. Un $4 \%$ del total de casos ha finalizado en la creación de una empresa a pequeña escala.

4. Los métodos de enseñanza con participación de los estudiantes, donde la responsabilidad del aprendizaje depende directamente de su actividad, implicación y compromiso son más formativos que meramente informativos, generan aprendizajes más profundos, significativos y duraderos y facilitan la transferencia a contextos más heterogéneos.

\section{Referencias}

ALCOBA, J. (2012). "La clasificación de los métodos de enseñanza en educación superior" en Contextos educativos: Revista de educación, 15, pp. 93-106.

COLÁS, M.P. (2005). "La formación universitaria en base a competencias" en Colás M.P. y De Pablo, J. "La universidad en la Unión Europea”. Málaga: Ed. Aljibe pp. 101-123.

DOMINGO, X. (1981). "La mesa del Buscón: en homenaje a Don Francisco de Quevedo y Villegas con ocasión de su centenario”. Ed. Tusquets. $181 \mathrm{pp}$.

FERNÁNDEZ MARCH, A. (2006). "Metodologías activas para la formación de competencias" en Educatio Siglo XXI, 24, pp. 35-56.

GONZÁLEZ, M.D.P. y MARTÍNEZ, E.M. (2008). "El profesor creativo y el profesor que potencia la creatividad en el contexto universitario" en Innovación Educativa, 18, pp. 203-211.

IBARRA, M.S. y RODRÍGUEZ, G. (2010). “Aproximación al discurso dominante sobre la evaluación del aprendizaje en la universidad” en Revista de educación, 351, pp. 385-407.

RIESCO, M. y DÍAZ, M. (2007). "La revisión entre iguales como herramienta de aprendizaje y evaluación en la asignatura de sistemas operativos" en XIII Jornadas de Enseñanza Universitaria de la Informática (2007. Teruel). Teruel: Universidad de Zaragoza. pp. 277-284.

STEVENS, D.D. y LEVI, A.J. (2013). "Introduction to rubrics: An assessment tool to save grading time, convey effective feedback, and promote student learning”. Ed. Stylus Publishing, LLC. 232 pp.

VILLARDÓN, L. (2006). "Evaluación del aprendizaje para promover el desarrollo de competencias" en Educatio Siglo XXI, 24, pp. 57-76.

WEISS, V.B. (1981). Citado por USSEGLIO-TOMASSET, L. 1998. "Métodos objetivos de valoración de los caracteres organolépticos" en Química Enológica. Ed. Mundi-Prensa. Madrid, pp. 381-400.

(c)) EY-NC-ND 2016, Universitat Politècnica de València

Congreso In-Red (2016) 